\title{
Sustaining community event-based surveillance in Sierra Leone
}

\author{
Eilidh M. Higgins*, Erin Polich, Maitreyi Sahu, Stacey Mearns and Ruwan Ratnayake \\ International Rescue Committee, Freetown, Sierra Leone
}

\section{Objective}

To assess whether the change in death swabbing policy in Sierra Leon has begun to affect community death reporting, we analyzed trends in death reporting before and after the policy change.

\section{Introduction}

Stemming from the 2014-6 Ebola virus disease (EVD) outbreak, community event based surveillance (CEBS) was implemented in Sierra Leone using community health workers to generate alerts for trigger events suggestive of EVD transmission. Through September 30, 2015 (last month of active EVD transmission), the majority (86\%) of alerts reflected community deaths; this was beneficial as Ebolarelated deaths were detected with delay during the epidemic's peak. The Government had implemented a policy of mandatory swabbing and testing of all dead bodies. The policy changed on June 30, 2016 wherein only swabbing of deaths deemed to be high-risk for EVD is required. To assess whether this policy change has begun to affect community death reporting, we analyzed trends in death reporting before and after the policy change.

\section{Methods}

This analysis was conducted using data from nine districts during period 1 (January-June 2016) and period 2 (July 2016). Weekly changes in the reporting of death alerts during the two periods were assessed. An interrupted time series analysis (ITS) with a segmented linear regression was also used to assess the immediate impact of the policy change.

\section{Results}

During period 1, monthly changes in death alerts across districts were variable $(-8 \%$ to $16 \%)$. Comparison of the weekly average between periods 1 and 2 showed a 33\% reduction in death alerts. During period 1 (before the policy change), there was an overall significant increase of 3.2 death alerts per week $(p=0.00)$ and no immediate impact or changes in the trend afterwards. At the district level, on average 354 death alerts were generated weekly in June, compared to 237 in July (33\% reduction); Moyamba district experienced the largest drop in death alerts from 46 to $16(65 \%)$.

\section{Conclusions}

Community death reporting provides early warning of EVD transmission by rapidly capturing death alerts where vital registration is not fully functional. Although we have one month of data postpolicy change, this preliminary analysis suggests that the change in swabbing policy may have halted an observed increase in death reporting. Further community mobilization efforts and training are warranted to prevent a drop in death reporting.

\section{Keywords}

Ebola; Surveillane; Community-Based

\section{Acknowledgments}

Many thanks to the UK's Department for International Development and the United Staes Agency for International Development.

\section{*Eilidh M. Higgins}

E-mail: eilidh.higgins@rescue.org 